\title{
Prevalência de alto risco para a síndrome da apneia obstrutiva do sono na população idosa residente na área rural de Rio Grande-RS
}

\section{High-risk prevalence for obstructive sleep apnea syndrome in elderly population resident in the rural area of Rio Grande-RS}

\author{
Aline Henriques Perceval ${ }^{* *}$ (D), Rodrigo Dalke Meucci' (D) \\ ${ }^{1}$ Faculdade de Medicina, Universidade Federal do Rio Grande (FURG) - Rio Grande (RS), Brasil.
}

Como citar: Perceval AH, Meucci RD. Prevalência de alto risco para a síndrome da apneia obstrutiva do sono na população idosa residente na área rural de Rio Grande-RS. Cad Saúde Colet, 2020;28(2):241-250. https://doi.org/10.1590/1414$462 X 202028020189$

\begin{abstract}
Resumo
Introdução: A população idosa tende a aumentar no Brasil com o passar dos anos. Dentre os problemas crônicos de saúde associados ao envelhecimento, destaca-se a Síndrome da Apneia Obstrutiva do Sono (SAOS). Objetivo: Verificar a prevalência do alto risco para a SAOS e seus fatores associados na população idosa residente na área rural do município de Rio Grande. Método: Estudo transversal, de base populacional com amostragem aleatória. Para a categorização do desfecho, foi utilizado o Questionário de Berlim, que consiste em avaliar a SAOS em Baixo e Alto Risco, e as variáveis independentes foram coletadas por meio de questionário padronizado. Resultados: Foram entrevistados 1.030 idosos. A prevalência do alto risco de SAOS foi de $38,4 \%$. Na análise ajustada, o relato de alto risco de SAOS foi maior em mulheres RP 1,18 (IC95\%1,01-1,38), idosos de 60-69 anos RP 1,78 (1,32-1,38), indivíduos que residiam com companheiro RP 1,28 (IC95\%1,06-1,53), diabéticos RP 1,27 (IC95\%1,05-1,53), que possuíam doença pulmonar RP 1,47 (IC95\%1,16-1,86) e que tiveram acidente vascular cerebral RP 1,36 (IC95\%1,07-1,76). Conclusão: Os achados desta pesquisa inferem que a prevalência elevada da SAOS na população idosa residente na área rural de Rio Grande, está associada a diversos outros fatores.
\end{abstract}

Palavras-chave: síndrome da apneia do sono; idosos de 80 anos ou mais; idoso; população rural.

\begin{abstract}
Background:The elderly population tends to increase in Brazil over the years. Among the chronic health issues associated with aging, it is pointed out the Obstructive Sleep Apnea Syndrome (OSAS). Objective: To verify high risk prevalence for OSAS and its associated factors in the elderly population living in the rural area of the municipality of Rio Grande. Method: A cross-sectional population-based study with random sampling was carried out. The Berlin Questionnaire was used to categorize the outcome, which consists of assessing a low- and high-risk OSAS, and the independent variables were collected using a standardized questionnaire. Results: 1,030 elderly people were interviewed. The prevalence of high risk of OSAS was $38.4 \%$. In the adjusted analysis or related to the high risk of OSAS; it was higher in women PR 1.18 (95\% Cl 1.01-1.38); elderly people aged 60 to 69 years PR 1.78 (1.32-1.38); individuals residing with a partner PR 1.28 (95\% Cl 1.06-1.53); diabetics individuals PR 1.27 (95\% Cl 1.05-1.53); individuals who had lung disease PR $1.47(95 \% \mathrm{Cl} 1), 16-1.86)$ and individuals who had cerebrovascular accident PR 1.36 (95\% CI 1.07-1.76). Conclusion: The results of this research infer that the high prevalence of OSAS in the elderly population living in the rural area of Rio Grande is associated with several other factors.
\end{abstract}

Keywords: sleep apnea syndromes; aged 80 and over; aged; rural population.

Trabalho realizado na área rural do município de Rio Grande (RS), Brasil.

Correspondência: Aline Henriques Perceval. E-mail: alinehenperceval@gmail.com

Fontes de financiamento: CNPQ, CAPES, Pastoral da Criança.

Conflito de interesses: nada a declarar.

Recebido em: Abr. 22, 2018. Aceito em: Jun. 25, 2019 


\section{INTRODUÇÃO}

A população idosa no Brasil tende a aumentar de 19,6 milhões em 2010 para 66,5 milhões em 2050, passando de $10 \%$ para cerca de $30 \%$. É prevista uma inversão na pirâmide populacional brasileira, sendo que os idosos com mais de 65 anos terão maior peso do que as crianças de 0-14 anos. Os pesquisadores têm cada vez mais se preocupado com esta população em específico devido ao grande aumento das doenças crônicas que estão relacionadas ao envelhecimento'.

Dentre os problemas crônicos de saúde associados ao envelhecimento, destaca-se a Síndrome da Apneia Obstrutiva do Sono (SAOS), a qual caracteriza-se por repetidas obstruções da via aérea superior que podem ser totais, apneias, ou parciais, hipopneias ${ }^{2}$. A SAOS pode levar a diversas morbidades, como isquemia, hipertensão, Acidente Vascular Cerebral (AVC), depressão, insônia, ansiedade e doença cardíaca coronariana. Além disso, pode levar à demência e a distúrbios na função cerebral, e causar acidentes de trânsito e de trabalho devido à sonolência diurna excessiva ${ }^{3-9}$.

O padrão ouro para o diagnóstico da SAOS é a polissonografia (PSG) convencional de noite inteira. Devido ao alto custo e dificuldade logística para utilização da PSG em estudos de base populacional, foram desenvolvidos questionários baseados em sinais e sintomas para rastreamento desta síndrome. Para tentar minimizar este problema, Netzer e seus colaboradores, em 1999, desenvolveram um questionário denominado "Questionário de Berlim" (QB), baseado em sinais e sintomas, que classificam os indivíduos em alto e baixo risco para SAOS ${ }^{10}$.

Em adultos de ambos os sexos, a prevalência de SAOS diagnosticada através de polissonografia varia de 7,5\% a 33\% $\%^{7,11}$. Quando utilizado o $\mathrm{QB}$, a prevalência do alto risco de SAOS vai de $5 \%$ a $30 \%{ }^{12-14}$. Em idosos, a prevalência da SAOS também apresenta grande variação, sendo encontrados estudos que relatam prevalências de 4,2\% a 46\% 4,15 .

Em áreas rurais, praticamente inexistem estimativas sobre a ocorrência de SAOS entre idosos. Foram encontrados apenas dois estudos, na China e na Turquia, que incluíram adultos e idosos de áreas rurais. Entretanto, a prevalência de SAOS especificamente por faixa etária não foi relatada, de modo que não é possível saber sua carga entre os idosos ${ }^{16,17}$. Especialmente no Brasil, não foram encontrados estudos de base populacional que avaliem a SAOS em área rural tanto para idosos quando para adultos. Assim, há uma lacuna no conhecimento deste desfecho em populações que moram afastadas dos grandes centros urbanos.

Deste modo, este estudo tem por objetivo avaliar a prevalência do alto risco de SAOS e seus fatores associados na população idosa residente na área rural do município de Rio Grande-RS no ano de 2017.

\section{MÉTODO}

Estudo transversal de base populacional, que faz parte de um consórcio de pesquisa do curso de mestrado do Programa de Pós-Graduação em Saúde Pública (PPGSP) da Universidade Federal do Rio Grande (FURG) denominado "Saúde da População Rural Rio-grandina", realizado de abril a outubro de 2017. O objetivo desta pesquisa era conhecer os indicadores básicos de saúde, o padrão de morbidade e a utilização dos serviços de saúde em crianças menores de cinco anos e suas mães, mulheres em idade fértil ( 15 a 49 anos) e idosos (60 anos ou mais).

Para o cálculo do tamanho amostral, utilizou-se uma prevalência estimada do alto risco de SAOS de $40 \%$, nível de confiança de $95 \%$, margem de erro de 3 pontos percentuais e acréscimo de $10 \%$ para perdas e recusas. Para o estudo dos fatores associados, foram utilizados os seguintes parâmetros: nível de confiança de $95 \%$, poder estatístico de $80 \%$, razão não expostos/expostos variando entre 45:55 e 94:6 e razões de prevalência entre 1,5 e 2,0. Ao tamanho de amostra calculado, foram adicionados mais $15 \%$ para controle de confundimento. De acordo com estes parâmetros, o maior tamanho de amostra necessário para este estudo seria de 844 indivíduos.

A área rural do município de Rio Grande é constituída por 24 setores censitários com cerca de 8.500 habitantes distribuídos em aproximadamente 2.700 domicílios permanentemente habitados. Para a seleção da amostra, utilizou-se um processo de amostragem de modo a 
selecionar $80 \%$ dos domicílios. Este processo foi realizado através do sorteio de um número entre " 1 " e " 5 ", sendo que o número sorteado correspondeu ao domicílio considerado pulo. Por exemplo, no caso do número " 3 " ter sido sorteado, todo domicílio de número " 3 " de uma sequência de cinco domicílios não era amostrado, ou seja, era pulado. Este procedimento garantiu que fossem amostrados quatro em cada cinco domicílios. Todos os idosos moradores dos domicílios amostrados foram considerados elegíveis e, portanto, convidados a participar do estudo. Foram incluídos na pesquisa idosos (60 anos ou mais) residentes na área rural do município de Rio Grande-RS. Quando um idoso era incapaz de responder ao questionário, a entrevista era realizada com um cuidador. Idosos hospitalizados e/ou privados de liberdade foram considerados inelegíveis para estudo.

Foi utilizado um questionário que avaliou aspectos socioeconômicos e demográficos (sexo, idade, estado civil e escolaridade), aspectos relacionados a saúde física, (diabetes, doenças pulmonares e AVC) e comportamentais (consumo de álcool e tabagismo). A coleta de dados foi realizada por entrevistadoras previamente treinadas e selecionadas. Os questionários eletrônicos foram aplicados por meio de tablets utilizando o programa RedCap ${ }^{\circledR 18}$.

Para a caracterização da variável dependente, foi utilizado o QB que classifica os indivíduos em baixo ou alto risco de SAOS através de 11 perguntas agrupadas em 3 categorias (Figura 1). A primeira compreende cinco questões referentes ao ronco e apneias durante o
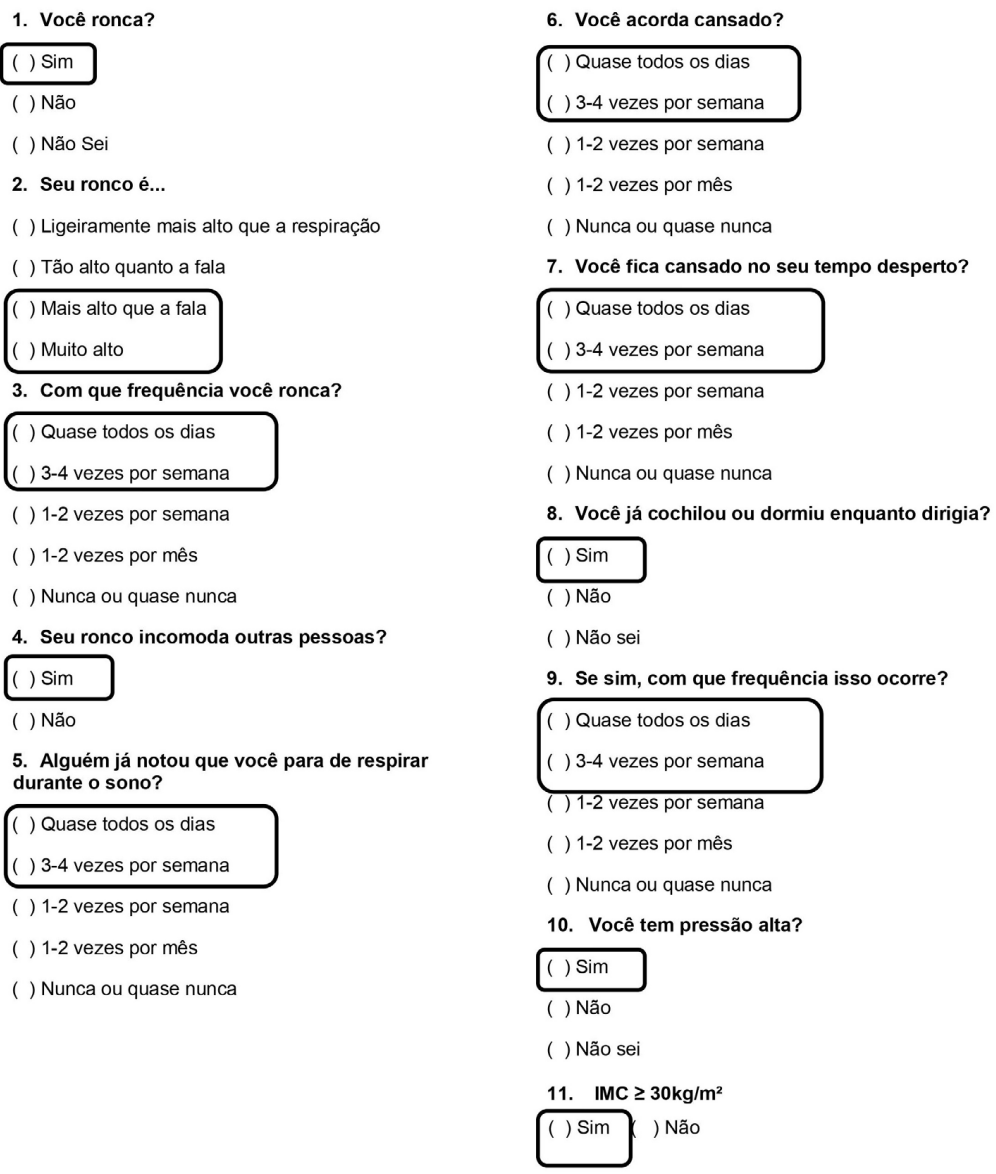

Alto Risco: é considerado alto risco a presença de duas ou mais categorias positivas

Categoria 1: presença de ronco com pelo menos uma das seguintes condições: mais alto do que a fala, pelo menos 3 a 4 vezes por semana, o ronco incomodar outras pessoas, pausas respiratórias testemunhadas por pelo menos 3 a 4 vezes por semana.

Categoria 2: acordar cansado pelo menos 3 a 4 vezes por semana, permanecer cansado durante o tempo desperto pelo menos 3 a 4 vezes por semana, adormecer ao volante

Categoria 3: IMC $\geq 30 \mathrm{kgm}^{2}$ ou médico ter relatado hipertensão

Figura 1. Classificação de Alto Risco de SAOS de acordo com o Questionário de Berlim 
sono. A segunda avalia a sonolência diurna excessiva, fadiga e cansaço e compreende quatro questões. A terceira categoria refere-se a hipertensão e obesidade. Será considerado como alto risco de apneia obstrutiva do sono se 2 das 3 categorias forem consideradas como positivas. Para isso, são necessários os seguintes critérios para cada uma das categorias: Categoria 1: presença de ronco com pelo menos uma das seguintes condições: mais alto do que a fala, pelo menos 3 a 4 vezes por semana, o ronco incomodar outras pessoas, pausas respiratórias testemunhadas por pelo menos 3 a 4 vezes por semana. Categoria 2: acordar cansado pelo menos de 3 a 4 vezes por semana, permanecer cansado durante o tempo desperto pelo menos de 3 a 4 vezes por semana, adormecer ao volante. Categoria 3: pressão arterial $\geq 140 / 90 \mathrm{mmHg}$ ou Obesidade (IMC $\geq 30 \mathrm{~kg} / \mathrm{m}^{2}$ ).

A análise de dados foi realizada no programa estatístico Stata versão $14^{\circledR}$. Inicialmente, as variáveis numéricas (idade e escolaridade) foram transformadas em categóricas ordinais e a variável situação conjugal coletada de forma categórica politômica foi operacionalizada como categórica dicotômica. Em seguida, realizou-se a análise descritiva que incluiu as frequências absolutas e relativas das variáveis independentes. Foram calculadas as proporções de cada categoria que compõem o desfecho em separado (Categoria 1, Categoria 2 e Categoria 3), seguidas da descrição da prevalência do desfecho de acordo com as variáveis independentes. Nesta etapa, foi utilizado o teste Quiquadrado de heterogeneidade.

Para exame dos fatores associados ao desfecho, foi utilizada Regressão de Poisson com ajuste robusto para variância nas análises bruta e ajustada. A significância estatística das associações foi verificada através do teste Wald de heterogeneidade para as exposições dicotômicas e politômicas e teste Wald de tendência linear para as exposições ordinais. A análise ajustada foi realizada utilizando o modelo hierárquico de análise definido a priori, constituído de quatro níveis. O primeiro nível foi constituído das variáveis sexo e idade. No segundo nível, foram inseridas as variáveis situação conjugal e escolaridade. No terceiro nível, tabagismo e uso de álcool nos últimos 30 dias, e, no quarto nível, diabetes, doença de pulmão e AVC ${ }^{19}$. Foi utilizada seleção para trás e considerado um valor $p<0,20$ para manter a variável no modelo e um $\mathrm{p}<0,05$ para as variáveis serem consideradas associadas ao desfecho.

O presente estudo foi analisado e aprovado pelo Comitê de Ética em Pesquisa da Universidade Federal do Rio Grande sob o parecer № 51/2017, processo 23116.009484/2016-26. Todos os idosos assinaram o Termo de Consentimento Livre e Esclarecido.

\section{RESULTADOS}

Foram amostrados 1.785 domicílios e identificados 1.131 idosos e, destes, 1.030 foram entrevistados, totalizando $8,9 \%$ de perdas e recusas. Na amostra estudada, predominavam homens $(55,1 \%)$, aproximadamente $52 \%$ possuíam idade entre 60 e 69 anos e $62 \%$ eram casados ou possuíam companheiro. Em relação à escolaridade, a maioria tinha até quatro anos de estudo. Cerca de 13\% eram fumantes, $16,7 \%$ consumiram álcool nos últimos 30 dias, 15,7\% eram diabéticos, 6,8\% possuíam algum tipo de doença pulmonar, 54,8\% eram hipertensos e aproximadamente $7 \%$ tiveram AVC (Tabela 1).

Os sintomas da categoria 1 foram mais frequentes nos indivíduos de 60-69 anos, que viviam com companheiro e hipertensos. Os sintomas na categoria 2 foram mais frequentes em mulheres, naqueles com 60-69 anos e nos que possuíam doença pulmonar. Sintomas da categoria 3 foram mais frequentes em mulheres, naqueles que nunca fumaram, não beberam nos últimos 30 dias, diabéticos, hipertensos e que relataram já ter tido AVC. A prevalência de alto risco de SAOS foi de $36 \%$ nos homens e $41,6 \%$ nas mulheres, entretanto esta diferença não foi estatisticamente significativa. A prevalência do desfecho foi maior naqueles com 60-69 anos, que possuíam companheiro, diabéticos, com algum tipo de doença pulmonar, hipertensos e naqueles que relataram ter tido AVC (Tabela 2).

Após análise ajustada, ser do sexo feminino RP 1,18 (IC95\% 1,01-1,38), ter 60-69 anos RP 1,78 (IC 95\% 1,32-1,38), viver com companheiro RP 1,28 (IC 95\% 1,06-1,53), ser diabético RP 1,27 (IC 95\% 1,05-1,53), ter doença pulmonar RP 1,47 (IC95\% 1,16-1,86) e ter tido AVC RP 1,36 (IC95\% 1,07-1,76) permaneceram associados ao desfecho (tabela 3). 
Tabela 1. Descrição das características da amostra de idosos da área Rural de Rio Grande, Rio Grande do Sul, Brasil, 2017 ( $\mathrm{N}=1030)$

\begin{tabular}{|c|c|c|}
\hline & $\mathbf{N}$ & $\%$ \\
\hline \multicolumn{3}{|l|}{ Sexo (1030) } \\
\hline Masculino & 568 & 55,1 \\
\hline Feminino & 462 & 44,9 \\
\hline \multicolumn{3}{|l|}{ Idade (1029) } \\
\hline 60 a 69 anos & 529 & 51,4 \\
\hline 70 a 79 anos & 326 & 31,8 \\
\hline 80 anos ou mais & 173 & 16,8 \\
\hline \multicolumn{3}{|l|}{ Situação Conjugal (1027) } \\
\hline Sem Complanheiro (a) & 390 & 37,9 \\
\hline Com companheiro (a) & 640 & 62,1 \\
\hline \multicolumn{3}{|l|}{ Escolaridade (1017) } \\
\hline 0-4 anos & 703 & 69,1 \\
\hline 5-11 anos & 247 & 24,3 \\
\hline 12 anos ou mais & 67 & 6,6 \\
\hline \multicolumn{3}{|l|}{ Tabagismo (1029) } \\
\hline Não, nunca fumou & 545 & 53,0 \\
\hline Já fumou, mas parou & 348 & 33,8 \\
\hline Sim, fuma & 136 & 13,2 \\
\hline \multicolumn{3}{|c|}{ Consumo de Álcool nos últimos 30 dias (1030) } \\
\hline Não & 858 & 83,3 \\
\hline Sim & 172 & 16,7 \\
\hline \multicolumn{3}{|l|}{ Diabetes (1028) } \\
\hline Não & 867 & 84,3 \\
\hline Sim & 161 & 15,7 \\
\hline \multicolumn{3}{|l|}{ Doença Pulmonar (1028) } \\
\hline Não & 958 & 93,2 \\
\hline Sim & 70 & 6,8 \\
\hline \multicolumn{3}{|l|}{ Hipertensão (1029) } \\
\hline Não & 465 & 45,2 \\
\hline Sim & 564 & 54,8 \\
\hline \multicolumn{3}{|l|}{ AVC (1030) } \\
\hline Não & 954 & 92,6 \\
\hline Sim & 76 & 7,4 \\
\hline
\end{tabular}

AVC: acidente vascular cerebral. Nota: a variável com maior número de valores ignorados foi a escolaridade ( $N=13)$ 
Tabela 2. Análise da prevalência das categorias que compõem o desfecho e o Alto Risco de SAOS conforme as variáveis independentes em uma amostra de idosos residentes na área rural do município de Rio Grande, Rio Grande do Sul, Brasil, 2017 ( $\mathrm{N}=1030)$

\begin{tabular}{|c|c|c|c|c|c|c|c|c|}
\hline & \multicolumn{2}{|c|}{ Categoria 1} & \multicolumn{2}{|c|}{ Categoria 2} & \multicolumn{2}{|c|}{ Categoria 3} & \multicolumn{2}{|c|}{ Alto Risco } \\
\hline & $\begin{array}{c}\text { Positivo } \\
\text { (\%) }\end{array}$ & $\mathbf{P}$ & $\begin{array}{c}\text { Positivo } \\
\text { (\%) }\end{array}$ & $\mathbf{P}$ & $\begin{array}{c}\text { Positivo } \\
\text { (\%) }\end{array}$ & $\mathbf{P}$ & $(\%)$ & $\mathbf{P}$ \\
\hline Sexo & & 0,78 & & 0,033 & & 0,000 & & 0,07 \\
\hline Masculino & 55,4 & & 5,3 & & 56,8 & & 36,0 & \\
\hline Feminino & 55,4 & & 8,7 & & 69,9 & & 41,6 & \\
\hline Faixa etária & & 0,000 & & 0,043 & & 0,35 & & $<0,0001$ \\
\hline 60 a 69 anos & 62,4 & & 8,7 & & 60,4 & & 42,2 & \\
\hline 70 a 79 anos & 55,8 & & 5,2 & & 64,9 & & 39,3 & \\
\hline 80 ou mais & 34,4 & & 4,1 & & 64,8 & & 23,8 & \\
\hline Estado civil & & 0,000 & & 0,150 & & 0,33 & & 0,001 \\
\hline Sem Companheiro (a) & 42,5 & & 8,3 & & 60,7 & & 31,4 & \\
\hline Com Companheiro(a) & 62,8 & & 5,9 & & 63,8 & & 42,4 & \\
\hline Escolaridade & & 0,591 & & 0,319 & & 0,42 & & 0,864 \\
\hline 0-4 anos & 55,1 & & 7,7 & & 63,3 & & 38,1 & \\
\hline 5-11 anos & 57,3 & & 5,3 & & 63,2 & & 40,0 & \\
\hline 12 anos ou mais & 61,3 & & 4,5 & & 55,2 & & 39,4 & \\
\hline Tabagismo & & 0,296 & & 0,764 & & 0,000 & & 0,290 \\
\hline Não, nunca fumou & 53,4 & & 7,4 & & 67,5 & & 38,0 & \\
\hline Já fumou, mas parou & 58,6 & & 6,4 & & 61,1 & & 41,1 & \\
\hline Sim, fuma & 58,1 & & 5,9 & & 46,6 & & 33,3 & \\
\hline $\begin{array}{l}\text { Consumo de Álcool nos } \\
\text { últimos } 30 \text { dias }\end{array}$ & & 0,712 & & 0,057 & & 0,000 & & 0,084 \\
\hline Não & 55,6 & & 7,5 & & 64,9 & & 39,7 & \\
\hline Sim & 57,1 & & 3,5 & & 51,7 & & 32,5 & \\
\hline Diabetes & & 0,237 & & 0,298 & & 0,000 & & 0,001 \\
\hline Não & 55,0 & & 6,5 & & 59,2 & & 36,2 & \\
\hline Sim & 60,3 & & 8,7 & & 80,1 & & 50,6 & \\
\hline Doença Pulmonar & & 0,390 & & 0,000 & & 0,078 & & 0,003 \\
\hline Não & 55,4 & & 5,6 & & 61,8 & & 37,1 & \\
\hline Sim & 60,9 & & 24,6 & & 72,5 & & 57,2 & \\
\hline Hipertensão & & 0,036 & & 0,254 & & 0,000 & & $<0,0001$ \\
\hline Não & 52,1 & & 5,8 & & 15,3 & & 12,4 & \\
\hline Sim & 59,0 & & 7,6 & & 100,0 & & 61,1 & \\
\hline AVC & & 0,103 & & 0,625 & & 0,003 & & 0,007 \\
\hline Não & 55,1 & & 6,7 & & 61,4 & & 37,3 & \\
\hline Sim & 65,2 & & 8,2 & & 78,7 & & 53,6 & \\
\hline
\end{tabular}

AVC: acidente vascular cerebral. Nota: a variável com maior número de valores ignorados foi a escolaridade ( $\mathrm{N}=13)$. Alto Risco: é considerado alto risco a presença de duas ou mais categorias positivas. Categoria 1: presença de ronco com pelo menos uma das seguintes condições: mais alto do que a fala, pelo menos 3 a 4 vezes por semana, o ronco incomodar outras pessoas, pausas respiratórias testemunhadas por pelo menos 3 a 4 vezes por semana. Categoria 2: acordar cansado pelo menos de 3 a 4 vezes por semana, permanecer cansado durante o tempo desperto pelo menos de 3 a 4 vezes por semana, adormecer ao volante. Categoria 3: pressão arterial $\geq 140 / 90 \mathrm{mmHg}$ ou Obesidade (IMC $\geq 30 \mathrm{~kg} / \mathrm{m}^{2}$ ) 
Tabela 3. Associação bruta e ajustada do Alto Risco de SAOS de acordo com as variáveis independentes em idosos residentes na área rural do município de Rio Grande, Rio Grande do Sul, Brasil, 2017 (N=1030)

\begin{tabular}{|c|c|c|c|c|}
\hline \multirow{2}{*}{ Variáveis } & \multicolumn{2}{|c|}{ Análise Bruta } & \multicolumn{2}{|c|}{ Análise Ajustada } \\
\hline & RP (IC95\%) & Valor $\mathbf{p}$ & RP (IC95\%) & Valor $p$ \\
\hline Sexo & & 0,07 & & 0,04 \\
\hline Masculino & 1 & & 1 & \\
\hline Feminino & $1,16(0,99-1,36)$ & & $1,18(1,01-1,38)$ & \\
\hline Faixa etária & & $0,0002 *$ & & $0,000 *$ \\
\hline 60 a 69 anos & $1,77(1,31-2,39)$ & & $1,78(1,32-1,38)$ & \\
\hline 70 a 79 anos & $1,65(1,21-2,25)$ & & $1,67(1,22-2,28)$ & \\
\hline 80 ou mais & 1 & & 1 & \\
\hline Estado civil & & 0,0012 & & 0,008 \\
\hline Sem Companheiro (a) & 1 & & 1 & \\
\hline Com Companheiro (a) & $1,35(1,13-1,62)$ & & $1,28(1,06-1,53)$ & \\
\hline Escolaridade & & $0,643^{*}$ & & $0,95^{*}$ \\
\hline $0-4$ anos & 1 & & 1 & \\
\hline 5-11 anos & $1,05(0,87-1,26)$ & & $1,01(0,84-1,22)$ & \\
\hline 12 anos ou mais & $1,03(0,76-1,42)$ & & $0,97(0,71-1,32)$ & \\
\hline Tabagismo & & 0,302 & & 0,193 \\
\hline Não, nunca fumou & 1 & & 1 & \\
\hline Já fumou, mas parou & $1,08(0,91-1,29)$ & & $1,13(0,94-1,36)$ & \\
\hline Sim, fuma & $0.88(0,67-1,14)$ & & $0,89(0,69-1,18)$ & \\
\hline Consumo de Álcool nos últimos 30 dias & & 0,097 & & 0,208 \\
\hline Não & 1 & & 1 & \\
\hline Sim & $0,82(0,65-1,04)$ & & $0,86(0,67-1,09)$ & \\
\hline Diabetes & & 0,0003 & & 0,012 \\
\hline Não & 1 & & 1 & \\
\hline Sim & $1,40(1,17-1,68)$ & & $1,27(1,05-1,53)$ & \\
\hline Doença Pulmonar & & 0,0008 & & 0.001 \\
\hline Não & 1 & & 1 & \\
\hline Sim & $1,49(1,17-1.87)$ & & $1,47(1,16-1,86)$ & \\
\hline AVC & & 0,0025 & & 0,013 \\
\hline Não & 1 & & 1 & \\
\hline Sim & $1,44(1,14-1,82)$ & & $1,36(1,07-1,76)$ & \\
\hline
\end{tabular}

*Teste $t$ de tendência linear. AVC: acidente vascular cerebral. Nota: a variável com maior número de valores ignorados foi a escolaridade $(\mathrm{N}=13)$ 


\section{DISCUSSÃO}

Este estudo mostrou uma elevada ocorrência de alto risco de SAOS (38,4\%), o qual está associado ao sexo feminino, ter de 60 a 79 anos, viver com companheiro, ter diabetes, doença pulmonar e ter tido AVC.

Em outros países, estudos que utilizaram o QB mostram que a prevalência do alto risco de SAOS em idosos de áreas urbanas tem grande variabilidade, sendo de $33 \%$ no $\operatorname{Iră}^{13}$, $21,2 \%$ na Colômbia ${ }^{20}$ e $46 \%$ na Jordânia ${ }^{15}$. No Brasil, um estudo realizado em Pelotas-RS com idosos residentes na área urbana que utilizou outro instrumento para caracterização da SAOS relata uma prevalência de $13,2 \%{ }^{21}$. Em São Paulo, a prevalência de SAOS mensurada por polissonografia foi de $60,2 \%$ e $87 \%$ para idosos de 60 a 69 e de 70 a 80 anos, respectivamente ${ }^{7}$

Embora na população adulta em geral seja reconhecido que o sexo masculino é um fator predisponente para SAOS $27,7,15,17,21,22$, neste estudo, as mulheres apresentaram maior risco para o desfecho. Isto pode ser decorrente do fato de que, no período pós-menopausa, a prevalência de SAOS tende a aumentar nas mulheres devido às mudanças hormonais. Além disso, com o envelhecimento, ocorre alongamento da faringe, o que aumenta o risco de desenvolver $\mathrm{SAOS}^{2,22}$.

O achado de que há maior risco do desfecho para idosos com 60 a 79 anos em relação àqueles com 80 anos ou mais é consistente com o relatado por Liu e seus colaboradores (2014), no qual idosos mais velhos (80 anos ou mais) possuem uma menor prevalência de SAOS em relação aos de menor idade. Estes achados podem ser decorrentes do viés de sobrevivência, pois idosos mais velhos são mais bem selecionados e, portanto, com menos doenças crônicas como a SAOS 17,23 .

Estudos demonstram que a diabetes está associada a uma respiração desordenada, o que ocasiona a SAOS ${ }^{4,11,24}$. Entretanto, esta associação pode ser bidirecional, pois a fragmentação do sono pode levar a efeitos adversos no metabolismo da glicose, que reduzem a sua captação. Há ainda, a possibilidade de que a diabetes pode piorar a gravidade da SAOS porque o aumento da glicose pode ser um fator de risco para hipoxemia noturna e aumento das apneias 22,25-27.

A variável doença pulmonar agrupou diversas patologias respiratórias, tais como Doença Pulmonar Obstrutiva Crônica (DPOC), asma e bronquite. Estas patologias muitas vezes apresentam mecanismos de ação semelhantes à SAOS. Idosos que possuem outras doenças pulmonares podem manifestar a SAOS com maior frequência do que aqueles que não possuem doenças pulmonares ${ }^{15,17}$.

A associação entre AVC e alto risco de SAOS é descrita na literatura, porém, por se tratar de um estudo transversal, não é possível estabelecer a temporalidade da associação. Isso ocorre devido ao fato de a SAOS participar de fatores como hipertensão, decréscimo do fluxo sanguíneo cerebral decorrente do menor débito cardíaco e hipercoagulabilidade sanguínea².

Apesar de o tabagismo ser um fator fortemente associado à SAOS em adultos ${ }^{4,17,21}$, neste estudo, esta associação não foi encontrada. É possível que, em idosos, esta associação não ocorra porque a prevalência de tabagismo tende a diminuir com a idade, principalmente entre os grupos que desenvolvem doenças crônicas ${ }^{28,29}$.

Embora seja um instrumento utilizado em estudos brasileiros ${ }^{7,30-32}$, o QB foi validado apenas em Portugal ${ }^{33}$. Deste modo, ainda são necessários estudos de validação deste instrumento para o português brasileiro. Existem poucos estudos que avaliem o alto risco de SAOS, utilizando o QB, em idosos, o que dificulta a comparabilidade com outros estudos. Salientamos que este estudo é inédito e identificou a prevalência do alto risco de SAOS em idosos de uma área rural, bem como seus fatores associados. Estes achados podem contribuir com os serviços de saúde, uma vez que apontam os grupos de idosos que devem ser preferencialmente rastreados para o alto risco de SAOS, sobretudo aqueles com doença pulmonar, diabetes e com história prévia de AVC.

\section{REFERÊNCIAS}

1. Instituto Brasileiro de Geografia e Estatística. Pesquisa Nacional de Saúde. Rio de Janeiro: IBGE; 2013.

2. Tufik S. Medicina e biologia do sono. 1. ed. Barueri: Manole; 2008. 483 p. 
3. Kamil MA, Teng CL, Hassan SA. Snoring and breathing pauses during sleep in the Malaysian population Respirology. 2007;12(3):375-80. http://dx.doi.org/10.1111/j.1440-1843.2007.01030.x. PMid:17539841.

4. Fuhrman C, Fleury B, Nguyen XL, Delmas MC. Symptoms of sleep apnea syndrome: high prevalence and underdiagnosis in the French population. Sleep Med. 2012;13(7):852-8. http://dx.doi.org/10.1016/j. sleep.2012.04.005. PMid:22705245.

5. Franklin KA, Sahlin C, Stenlund H, Lindberg E. Sleep apnoea is a common occurrence in females. Eur Respir J. 2013;41(3):610-5. http://dx.doi.org/10.1183/09031936.00212711. PMid:22903961.

6. Sands-Lincoln M, Grandner M, Whinnery J, Keenan BT, Jackson N, Gurubhagavatula I. The association between obstructive sleep apnea and hypertension by race/ethnicity in a nationally representative sample. J Clin Hypertens. 2013;15(8):593-9. http://dx.doi.org/10.1111/jch.12144. PMid:23889723.

7. Tufik S, Santos-Silva R, Taddei JA, Bittencourt LR. Obstructive sleep apnea syndrome in the Sao Paulo Epidemiologic Sleep Study. Sleep Med. 2010;11(5):441-6. http://dx.doi.org/10.1016/j.sleep.2009.10.005. PMid:20362502.

8. Ancoli-Israel S, Klauber MR, Butters N, Parker L, Kripke DF. Dementia in institutionalized elderly: relation to sleep apnea. J Am Geriatr Soc. 1991;39(3):258-63. http://dx.doi.org/10.1111/j.1532-5415.1991.tb01647.x. PMid:2005339.

9. Phillipson EA. Sleep apnea-a major public health problem. N Engl J Med. 1993;328:1271-73. http://dx.doi. org/10.1056/NEJM199304293281712.

10. Netzer NC, Stoohs RA, Netzer CM, Clark K, Strohl KP. Using the Berlin Questionnaire to identify patients at risk for the sleep apnea syndrome. Ann Intern Med. 1999;131(7):485-91. http://dx.doi.org/10.7326/00034819-131-7-199910050-00002. PMid:10507956.

11. Pływaczewski R, Bednarek $M$, Jonczak L, Zielinski J. Sleep-disordered breathing in a middle-aged and older Polish urban population. J Sleep Res. 2008;17(1):73-81. http://dx.doi.org/10.1111/j.13652869.2008.00632.x. PMid:18275557.

12. Hiestand DM, Britz P, Goldman M, Phillips B. Prevalence of symptoms and risk of sleep apnea in the US population: Results from the national sleep foundation sleep in America 2005 poll. Chest. 2006;130(3):7806. http://dx.doi.org/10.1378/chest.130.3.780. PMid:16963675.

13. Amra B, Farajzadegan Z, Golshan M, Fietze I, Penzel T. Prevalence of sleep apnea-related symptoms in a Persian population. Sleep \& Breath. 2011;15(3):425-9.

14. Khazaie H, Najafi F, Rezaie L, Tahmasian M, Sepehry AA, Herth FJ. Prevalence of symptoms and risk of obstructive sleep apnea syndrome in the general population. Arch Iran Med. 2011;14(5):335-8. PMid:21888458.

15. Khassawneh B, Ghazzawi M, Khader Y, Alomari M, Amarin Z, Shahrour B, et al. Symptoms and risk of obstructive sleep apnea in primary care patients in Jordan. Sleep \& Breath. 2009;13(3):227-32.

16. Ekici M, Ekici A, Keles $\mathrm{H}$, Akin A, Karlidag A, Tunckol M, et al. Risk factors and correlates of snoring and observed apnea. Sleep Med. 2008;9(3):290-6. http://dx.doi.org/10.1016/j.sleep.2007.04.018. PMid:17658298.

17. Liu J, Wei C, Huang L, Wang W, Liang D, Lei Z, et al. Prevalence of signs and symptoms suggestive of obstructive sleep apnea syndrome in Guangxi, China. Sleep Breath. 2014;18(2):375-82. http://dx.doi. org/10.1007/s11325-013-0896-2. PMid:24072550.

18. Harris PA, Taylor R, Thielke R, Payne J, Gonzalez N, Conde JG. Research electronic data capture (REDCap) A metadata-driven methodology and workflow process for providing translational research informatics support. J Biomed Inform. 2009;42(2):377-81. http://dx.doi.org/10.1016/j.jbi.2008.08.010. PMid:18929686

19. Victora CG, Huttly SR, Fuchs SC, Olinto MT. The role of conceptual frameworks in epidemiological analysis: a hierarchical approach. Int J Epidemiol. 1997;26(1):224-7. http://dx.doi.org/10.1093/ije/26.1.224. PMid:9126524.

20. Ruiz AJ, Sepúlveda MAR, Franco OH, Cepeda M, Martinez PH, Garzón SBA, et al. The associations between sleep disorders and anthropometric measures in adults from three Colombian cities at different altitudes. Maturitas. 2016;94:1-10. http://dx.doi.org/10.1016/j.maturitas.2016.08.013. PMid:27823726.

21. Noal RB, Menezes AMB, Canani SF, Siqueira FV. Ronco habitual e apnéia obstrutiva observada em adultos: estudo de base populacional, Pelotas, RS. Rev Saude Publica. 2008;42(2):224-33. http://dx.doi.org/10.1590/ S0034-89102008000200006. PMid:18372974.

22. Heinzer R, Vat S, Marques-Vidal P, Marti-Soler H, Andries D, Tobback N, et al. Prevalence of sleep-disordered breathing in the general population: the HypnoLaus study. Lancet Respir Med. 2015;3(4):310-8. http:// dx.doi.org/10.1016/S2213-2600(15)00043-0. PMid:25682233.

23. Gordis L. Epidemiologia. 4. ed. Rio de Janeiro: Revinter; 2008. 
24. Kent BD, Grote L, Ryan S, Pepin JL, Bonsignore MR, Tkacova R, et al. Diabetes mellitus prevalence and control in sleep-disordered breathing: the European Sleep Apnea Cohort (ESADA) study. Chest. 2014;146(4):98290. http://dx.doi.org/10.1378/chest.13-2403. PMid:24831859.

25. Aurora RN, Punjabi NM. Obstructive sleep apnoea and type 2 diabetes mellitus: a bidirectional association. Lancet Respir Med. 2013;1(4):329-38. http://dx.doi.org/10.1016/S2213-2600(13)70039-0. PMid:24429158.

26. Reutrakul S, Mokhlesi B. Obstructive sleep apnea and diabetes: a state of the art review. Chest. 2017;152(5):1070-86. http://dx.doi.org/10.1016/j.chest.2017.05.009. PMid:28527878.

27. Liu C-L, Wu C-S. Assessing whether the association between sleep apnea and diabetes is bidirectional. Can J Diabetes. 2017;41(2):197-203. http://dx.doi.org/10.1016/j.jcjd.2016.09.009. PMid:27989495.

28. Horta BL, Ramos EO, Victoria CG. Determinantes do hábito de fumar na cidade de Pelotas, Brasil. Bol Oficina Sanit Panam 1992;113:131-6.

29. Freitas ERFS, Ribeiro LRG, de Oliveira LD, Rissas JM, Domingues VI. Fatores associados ao tabagismo em idosos residentes na cidade de Londrina, Brasil. Rev Bras Geriatr Gerontol. 2010;13(2):277-87. http://dx.doi. org/10.1590/S1809-98232010000200012.

30. Hirotsu C, Tufik S, Guindalini C, Mazzotti DR, Bittencourt LR, Andersen ML. Association between uric acid levels and obstructive sleep apnea syndrome in a large epidemiological sample. PLoS One. 2013;8(6):1-9. http://dx.doi.org/10.1371/journal.pone.0066891. PMid:23826169.

31. Andrechuk CRS, Ceolim MF. Alto risco para síndrome da apneia obstrutiva do sono em pacientes com infarto agudo do miocárdio. Rev Lat Am Enfermagem. 2015;23(5):797-805. http://dx.doi.org/10.1590/01041169.0511.2617. PMid:26487128.

32. Koyama RG, Esteves AM, Silva LO, Lira FS, Bittencourt LR, Tufik S, et al. Prevalence of and risk factors for obstructive sleep apnea syndrome in Brazilian railroad workers. Sleep Med. 2012;13(8):1028-32. http:// dx.doi.org/10.1016/j.sleep.2012.06.017. PMid:22841037.

33. Vaz A, Drummond M, Mota PC, Severo M, Almeida J, Winck JC. Tradução do Questionário de Berlim para língua Portuguesa e sua aplicação na identificação da SAOS numa consulta de patologia respiratória do sono. Rev Port Pneumol. 2011;17(2):59-65. http://dx.doi.org/10.1016/S0873-2159(11)70015-0. PMid:21477567. 